\title{
The mechanics behind gait problems in patients with Dravet Syndrome
}

\section{Authors}

Lore Wyers, MSc, Research Group MOVANT, Department of Rehabilitation Sciences and Physiotherapy (REVAKI), University of Antwerp, Wilrijk, Belgium; Multidisciplinary Motor Centre Antwerp, University of Antwerp, Belgium; Department of Rehabilitation Sciences, KU Leuven, Leuven, Belgium

Karen Verheyen, MSc, Research Group MOVANT, Department of Rehabilitation Sciences and Physiotherapy (REVAKI), University of Antwerp, Wilrijk, Belgium; Multidisciplinary Motor Centre Antwerp, University of Antwerp, Belgium

Berten Ceulemans, MD, PhD, Department of Paediatrics, Antwerp University Hospital, University of Antwerp, Antwerp, Belgium

An-Sofie Schoonjans, MD, Department of Paediatrics, Antwerp University Hospital, University of Antwerp, Antwerp, Belgium

Kaat Desloovere, PhD, Department of Rehabilitation Sciences, KU Leuven, Leuven, Belgium; Clinical Motion Analysis Laboratory, University Hospital Leuven, Pellenberg, Belgium

Patricia Van de Walle*,1, PhD, Research Group MOVANT, Department of Rehabilitation Sciences and Physiotherapy (REVAKI), University of Antwerp, Wilrijk, Belgium; Multidisciplinary Motor Centre Antwerp, University of Antwerp, Belgium

Ann Hallemans*,1, PhD, Research Group MOVANT, Department of Rehabilitation Sciences and Physiotherapy (REVAKI), University of Antwerp, Wilrijk, Belgium; Multidisciplinary Motor Centre Antwerp, University of Antwerp, Belgium

${ }^{1}$ Patricia Van de Walle and Ann Hallemans equally contributed to this article as last author.

*Corresponding author at: Movant, Universiteitsplein 1, Campus Drie Eiken, D.S.022, Wilrijk, 2610, Belgium. E-mail address: ann.hallemans@uantwerpen.be (A. Hallemans).

\section{Reference}

Wyers L., Verheyen K., Ceulemans B., Schoonjans A-S., Desloovere K., Van de Walle P., et al. The mechanics behind gait problems in patients with Dravet Syndrome. Gait Posture 2020;84 (December 2020):321-8. Doi: 10.1016/j.gaitpost.2020.12.029.

\section{Abstract}

\section{Background}

Dravet Syndrome (DS) is a developmental and epileptic encephalopathy starting in infancy and characterised by treatment resistant epilepsy with cognitive impairment and progressive motor dysfunction. Walking becomes markedly impaired with age, but the mechanical nature of gait problems remains unclear.

\section{Research question}

What are the kinetic strategies characterised in gait of patients with DS? 


\section{Methods}

This case-control study compared 41 patients with DS aged 5.2 to 26.1 years (19 female, 22 male) to 41 typically developing (TD) peers. Three dimensional gait analysis (VICON) was performed to obtain spatiotemporal parameters, kinematics and kinetics during barefoot, level walking at self-selected walking velocity. The sagittal plane support moment was analysed using Statistical Parametric Mapping (SPM). Three DS subgroups were identified based on differences in kinetic strategies characterised by the net internal knee joint moments and trunk lean. Kinematic and kinetic time profiles of the subgroups were compared to the TD group (SPM $t$-test). Clinical characteristics from physical examination and parental anamnesis were compared between DS (sub)groups using non-parametric tests (Kruskal-Wallis, Wilcoxon rank-sum, Fisher's exact).

\section{Results}

Support moments in stance were significantly increased in the DS group compared to TD and strongly related to minimum knee flexion in midstance. Persistent internal knee extension moments during stance were detected in a subgroup of $27 \%$ of the patients. A second subgroup of $34 \%$ showed forward trunk lean and attained internal knee flexion moments. The remaining $39 \%$ had neutral or backward trunk lean with internal knee flexion moments. Subgroups differed significantly in age and functional mobility.

\section{Significance}

Inefficient kinetic patterns suggested that increased muscle effort was needed to control lower limb stability. Three distinct kinetic strategies that underly kinematic deviations were identified. Clinical evaluation of gait should pay attention to knee angles, trunk lean and support moments.

\section{Keywords}

Dravet Syndrome, Severe Myoclonic epilepsy of infancy (SMEI), kinematics, kinetics, support moment, trunk lean

\section{Highlights}

- This is the first study to document kinetics of gait in patients with Dravet Syndrome

- Lower limb support moments were increased in patients with Dravet Syndrome

- Three subgroups were characterised by sagittal knee moment and trunk lean

- Crouch gait and skeletal malalignments were detected

- Kinetics and trunk lean revealed distinct strategies behind kinematic deviations.

\section{Introduction}

Dravet Syndrome (DS) is a developmental and epileptic encephalopathy characterized by drug resistant infantile onset seizures with cognitive and progressive motor impairments [1,2]. It is primarily caused by mutations in the neuronal sodium voltage-gated channel type 1 alpha subunit encoding gene (SCN1A) [3]. The sodium channel interneuronopathy induces intractable epileptic seizures and a variety of comorbidities [4]. Around adolescence, walking problems become evident, making many of the patients lean on others or use a wheelchair for longer distances [5]. Literature on gait deviations in patients with DS is still scarce and mainly observational [6]. Quantitative analysis of gait in this population is only recently reported [7]. Crouch gait is often described in about half of the population, next to a variety of other deviations [6,7]. Crouch gait is defined by excessive knee flexion in stance and was originally described in populations with cerebral palsy, where it is caused by a 
complex of muscle weakness, spasticity and contractures [8,9]. However, these symptoms are rarely seen in patients with DS $[6,10]$, hence the nature of their gait problems remains unclear.

Studying biomechanics provides insight in how the central nervous system controls movements. The central nervous system can select many combinations of muscle forces to yield the same moment around a joint. Moreover, many combinations of hip, knee and ankle moments can result in the same knee angle. Therefore, when deviations in joint angles (kinematics) are observed, analysis of muscle and soft tissue forces that cause these motions (kinetics) is necessary to understand the neuromuscular control behind gait abnormalities [11].

To obtain an indicator of lower limb control during gait [12,13], Winter (1980) proposed to combine the three major lower limb moments into one single measure: the 'support moment' $\left(M_{S}\right)$, defined as the algebraic sum of the net internal extension moments at hip, knee and ankle [12]. The magnitude of the $M_{S}$ depends on the ground reaction force $(\mathrm{GRF})$ and the knee flexion angle and can be interpreted as the total internal extension moment that is generated to prevent collapse of the stance limb [13]. Relatively higher $M_{S}$ may thus suggest that extensor muscles are inducing larger moments and more muscular effort is needed to stabilise the limb.

To maintain stability of the stance limb in normal gait, ankle plantar flexors are active in midstance to slow down the forward momentum and align the GRF anterior to the knee. An internal hip and knee flexion moment produced by soft tissue forces is attained during single leg stance. This way, less muscle activity is required, which optimizes the energy expenditure [14]. When a person fails to align the GRF in front of the knee, persistent internal knee extension moments occur, greatly increasing energy demands [14]. Forward trunk lean influences the direction of the GRF and is often observed as a compensatory strategy to reduce this internal extension moment [15].

The observed gait deviations in patients with DS, especially wide based and crouch gait, may reflect a lack of stance limb stability [7], probably caused by underlying neuromuscular control disturbance [4,16,17]. Recently, Di Marco et al. (2019) reported gait kinematics of patients with DS [7], but to our knowledge, no studies on gait kinetics in DS have been published yet. Therefore, this study aims to characterise kinetic strategies employed by patients with DS to support the lower limb during stance phase of gait. First, analysis of the $M_{S}$ may provide evidence of lower limb support abnormalities. Furthermore, distinguishing subgroups based on differences in kinetic strategies characterised by the net internal knee joint moments and trunk lean, may enhance understanding of the heterogeneity of gait deviations. Lastly, detecting differences in gait and clinical characteristics between these subgroups may highlight the functional impact of the observed kinetic strategies.

\section{Methods}

\section{Study design and setting}

This case-control study was part of a larger project registered at ClinicalTrials.gov (https://clinicaltrials.gov/ct2/show/NCT03857451) and approved by the ethics committees of the Antwerp and Leuven University Hospitals (Belgian Registration Number: B300201627079). Patient data collection was performed between May 2016 and February 2020 (most recent session selected per patient) at the Multidisciplinary Motor Centre Antwerp (M²OCEAN).

\section{Participants}

Volunteers with DS were recruited through the department of child neurology at the Antwerp University Hospital and the parent organization of the Netherlands and Flanders 'Stichting Dravetsyndroom 
Nederland/Vlaanderen'. All candidates with a clinical diagnosis with DS were included if SCN1A mutation was confirmed and they had a minimum age of five and maximum of 25 years at enrolment. Exclusion criteria were the occurrence of a severe epileptic seizure within 24 hours before assessment and comorbidities of neurological and/or orthopaedic disorders not related to DS (figure 1).

\section{Data collection}

\section{Gait data}

Body segment motion and ground reaction forces were captured and processed using a VICON system (Nexus v2.8.1, VICON, Oxford Metrics, Oxford, UK) with eight optoelectronic cameras (100 Hz), two optical cameras (50 fps, Basler AG, Ahrensburg, Germany) and four force plates (1000 Hz, low-pass filtered at $20 \mathrm{~Hz}$, AMTI, Watertown, MA, US). Retroreflective markers were placed following the PlugInGait Lower Limb marker model [18]. All participants performed walking trials at self-selected walking velocity. Patients were only included when kinetic data were successfully collected. A control group, balanced for age, was selected from available databases of typically developing children and healthy adults without neurological or orthopaedic conditions, collected at $\mathrm{M}^{2} \mathrm{OCEAN}$ and the Clinical Motion Analysis Laboratory of the University Hospital Pellenberg, Leuven (CMAL-P), collected and processed with identical procedures. All gait analysis and physical examination data were collected by the same two researchers (L.W. and K.V., MSc physiotherapists)

\section{Clinical characteristics}

Via medical record screening and parental anamnesis, the following information was obtained: type of mutation, age of epilepsy onset, current epileptic severity and age of independent walking. The patients usual mobility was inquired using the Functional Mobility Scale (FMS) and by asking the maximum distance the patient was currently able to walk ('walking distance'). Note that we gave FMS score four when the patient usually held a person's hand [19]. Levels of intellectual disability (ID) were estimated as mild, moderate or severe, supported by cognitive test scores if available [20]. During physical examination, goniometric measures of joint range of motion (ROM), muscle length and skeletal alignment were obtained and compared to age-related norm values $[21,22]$.

\section{Data processing}

Spatiotemporal parameters, lower limb joint kinematics and kinetics were calculated. Visual inspection of data quality and further processing was performed using custom made MATLAB ${ }^{\circ}$ software (vR2018a, The Mathworks Inc, Natick, MA, US). Spatiotemporal and kinetic data were non-dimensionally normalized for leg length, body mass and gravitational acceleration [23,24]. For each instant of the gait cycle, sagittal plane $M_{S}$ were calculated according to the formula by Hof (2000) [13].

$M_{S}=\frac{M_{H}}{2}+M_{K}+\frac{M_{A}}{2}$

with $M_{H}, M_{K}$, and $M_{A}$ indicating the net internal moment (positive values for extension) around hip, knee and ankle respectively [13]. Per participant, all available trials of one randomly selected side (ranging from one to twelve trials) were averaged, because further analyses required single observations per subject.

Trunk lean was assessed by one researcher (L.W.) based on sagittal plane video images. The angle between vertical and the trunk axis (estimated trochanter major to acromion) was measured on a still frame taken at 'opposite toe-off' using Kinovea (v0.8.15, http://www.kinovea.org). Trunk lean was then categorized as 'neutral' (between vertical and $5^{\circ}$ of forward or backward inclination), 'forward' or 'backward' $[15,25]$. 


\section{Subgrouping}

We constructed a decision making tree to identify subgroups within DS based on minimal sagittal knee moment in midstance (between $30 \%$ of stance phase and toe-off) and trunk lean. The KMext subgroup was defined by a persistent internal knee extension moment throughout stance, regardless of trunk position. The KMflex-Tf subgroup contained patients who attained an internal knee flexion moment in midstance in combination with forward trunk lean. The remaining patients formed the KMflex-Tn/b subgroup, characterized by an internal knee flexion moment in combination with a neutral or backward inclined trunk (figure 1).

\section{Statistical analysis}

Spatiotemporal parameters and clinical characteristics were analysed using non-parametric tests, since graphical inspection and formal tests (Kolmogorov-Smirnov) revealed that the assumption of normality was not fulfilled. Wilcoxon rank-sum tests (two-tailed $\alpha=.05$ ) were used to compare age, BMI and non-dimensional spatiotemporal parameters between DS and TD. Fisher's exact tests (categorical data, including physical examination categorised as 'normal' or 'deviant') and Kruskal-Wallis tests (two-tailed, $\alpha=.05$ ) with post-hoc pairwise Wilcoxon rank-sum tests with Bonferroni correction (numerical data) were used to identify differences in clinical characteristics between subgroups. As Fisher's exact test is somewhat conservative [28], $\alpha$-levels were set at 0.10 for this test.

Kinematic and kinetic time profiles were analysed using statistical parametric mapping (SPM) [26]. Prior to SPM analyses, time profiles were normalized to $50 \%$ stance and $50 \%$ swing phase, in order to eliminate influence of differences in stance phase duration. Test statistics ( $t$-test or regression, see further) were calculated for each time node and expressed as $\operatorname{SPM}\{t\}$ trajectories. A critical threshold was then defined that only 5\% $(\alpha=0.05)$ of identically smooth random curves were expected to exceed. Parts of the gait cycle where SPM $\{t\}$ trajectory crossed this threshold were identified as clusters with significant outcome, for which cluster-specific $P$-values were calculated based on the Random Field Theory [27]. Small clusters ( $<3 \%$ GC) were not considered clinically relevant and therefore not discussed. An SPM $t$-test was used to identify differences in $M_{S}$ between DS and TD. SPM regression analyses explored association with walking velocity and minimum knee angle in midstance for DS and TD separately. After that, SPM $t$-tests were used to detect kinematic and kinetic deviations comparing each subgroup to the TD group. Bonferroni correction for multiple testing brought $\alpha$ to .017. All SPM analyses were performed using spm1d open source code (vM.0.4.5, http://www.spm1d.org) in MATLAB®. All other statistical analyses were executed in R (v4.0.0, R Foundation, Vienna, Austria)

\section{Results}

\section{Participants}

Out of 50 candidates, 41 patients aged 5.2 to 26.1 years (19 female, 22 male) were included in this study (individual characteristics: appendix, table A1). Five patients were not eligible and four others were not cooperative enough to collect kinetic data (figure 1). The control group of 41 TD individuals did not differ from the DS group for age and BMI. Participants with DS walked significantly slower with shorter and wider steps and longer relative stance time $(P<.05$; table 1$)$.

\section{Support moment}

SPM $t$-test showed a significantly higher $M_{S}(P<.001)$ in the DS group during most of stance phase (figure 2, left panel). Significant positive associations between $M_{S}$ and walking velocity were identified in early stance $(P<.001)$ only for the DS group. Significant positive associations $(P<.001)$ with minimum knee flexion angle in 
midstance were observed for the majority of stance for both groups (figure 2, middle panel). The absence of an association with walking velocity in midstance and the significant correlation with knee angle are illustrated for one time point (50\% stance phase) in figure 2, right panel. Details of SPM analyses can be consulted in appendix figure A1.

\section{Subgrouping}

Three DS subgroups were identified using the decision making tree. The KMext subgroup included eleven patients (27\%) of whom five walked with neutral trunk and six showed forward lean. The KMflex-Tf subgroup consisted of fourteen patients (34\%). The KMflex-Tn/b subgroup of sixteen patients (39\%) of whom seven had a neutral trunk position and nine a backward lean (figure 1).

\section{Gait pattern}

Comparison of kinematic and kinetic curves (internal moments) revealed significant differences for each subgroup compared to the TD group (figure 3).

In KMext, kinematics were characterized by significantly increased hip $(P<.001)$ and knee $(P<.001)$ flexion for most of the gait cycle, increased ankle dorsiflexion in mid- and terminal stance (MSt-TSt; $P<.001$ ), decreased ankle plantar flexion in initial swing (ISw; $P=.004)$ and increased external foot progression $(P<.001)$. The underlying $M_{S}$ was significantly increased in loading response (LR; $\left.P=.001\right)$ and MSt-TSt $(P<.001)$, resulting from decreased flexion moments at the hip in TSt $(P<.001)$, increased knee extension moments in MSt-TSt $(P<.001)$, ankle plantarflexion moments in LR $(P=.001)$ and decreased plantarflexion moments around TSt $(P<.001)$.

In KMflex-Tf, kinematics were characterized by increased anterior pelvic tilt $(P<.001)$, hip $(P<.001)$ and knee $(P<.001)$ flexion over the entire gait cycle, with increased dorsiflexion in LR $(P=.004)$ and MSt $(P=.016)$ and decreased dorsiflexion in pre-swing (PSw) and ISw $(P<.001)$ and increased external foot progression $(P<.001)$. The $M_{S}$ was significantly increased from LR to TSt $(P<.001)$, resulting from increased hip extension moments in LR $(P=.014)$ and MSt-TSt $(P<.001)$, knee flexion moments in LR $(P=.017)$ and TSt $(P<.001)$ and plantarflexion moments in LR $(P<.001)$ and decreased plantarflexion moment around TSt $(P<.001)$. Furthermore, first $(P=.002)$ and second $(P<.001)$ peak hip abduction moment were decreased.

In KMflex-Tn/b, kinematics were characterized by increased hip flexion over stance and ISw $(P<.001)$ and in terminal swing (TSw; $P=.016)$ and knee flexion from initial contact to TSt $(P<.001)$ and in TSw $(P=.011)$ and increased external foot progression $(P<.001)$. Ankle dorsiflexion was increased in LR $(P=.006)$, MSt $(P=.004)$, PSw $(P=.012)$ and ISw $(P<.001)$. Significantly increased $M_{S}$ were found in LR $(P=.002)$ and in MSt $(P<.001)$, resulting from decreased hip flexion moments in TSt $(P<.001)$, knee extension moments in LR $(P=.012)$ and MSt-TSt $(P<.001)$, and plantarflexion moments in TSt $(P=.001)$. Furthermore, second peak hip abduction moment was decreased $(P<.001)$.

In swing, significant differences in joint moments were also identified for each subgroup, but will not be discussed since they did not attribute to stance limb support. Additional graphs of coronal and transverse plane are reported in the appendix (figure A2). Details of SPM analyses can be consulted in the appendix, (figure A3).

\section{Clinical characteristics}

Subgroups differed significantly in age, walking distance and the FMS-500m scores. Post-hoc tests indicated that KMext contained older participants than KMflex-Tf, while patients in KMflex-Tn/b could walk longer distances and walked more independently (table 2). Abnormalities in ROM, muscle length and alignment were present in 
the three subgroups, equally distributed. Most frequent deviations were plantar flexor tightness, external tibial torsion and planovalgus feet (table 3 ). No clear spasticity was detected.

\section{Discussion}

This study aimed to characterise kinetic strategies in gait of patients with DS by evaluating the $M_{S}$ and defining subgroups based on internal knee extension moments and trunk lean. Increased $M_{S}$ were observed and three main strategies were identified with characteristic kinematic and kinetic deviations.

The $M_{S}$ equals force in the direction of the hip-ankle axis times knee eccentricity and is thus mainly determined by GRF magnitude and knee flexion angles [13]. Walking velocity is known to affect both factors and was significantly different between the DS and TD. The absence of an association in midstance, however, suggested that $M_{S}$ abnormalities were not just walking velocity effects. Knee flexion angles on the other hand were significantly increased in the three subgroups and correlated with $M_{S}$. The increased $M_{S}$ suggested that participants with DS require more muscular effort for stance limb support. Future studies on EMG activity should test this hypothesis. Three subgroups were distinguished based on strategies that could influence the knee eccentricity.

Although six patients in KMext walked with forward trunk lean, they all failed to align the GRF in front of the knee. The resulting kinematic pattern of flexion in hip, knee and ankle with a neutral pelvis position and persistent internal knee extension moment can be defined as 'uncompensated crouch gait' [9,29]. Persistent knee extension moments are expected to require extra muscle activity and thereby lead to higher energy costs that could impact functional mobility in this subgroup.

Even though KMflex-Tf also showed increased flexion in the three lower limb joints, the forward trunk lean strategy might reduce $M_{S}$. However, anterior pelvic tilt and hip flexion increased as a consequence and evoked increased internal hip extension moments, so the resulting $M_{S}$ remained high compared to TD. The required increased hip extensor activity makes this pattern energetically inefficient. The trunk and pelvis position together with internal knee flexion moments can be classified as 'compensated crouch gait' [29]. It remains unclear whether trunk lean was purely a support strategy or reflected underlying trunk control deficits [15].

In contrast to the other subgroups, internal knee flexion moments were attained without forward trunk lean in KMflex-Tn/b. The observation of normal internal plantar flexion moments in early- and midstance suggested functional plantar flexion - knee extension couple. However, $M_{S}$ were still increased compared to TD, combined with increased flexion angles in hip, knee and ankle. Nevertheless, all deviations remained close to normative values, indicating that gait in this subgroup was only mildly affected.

Our subgrouping process was based on kinetic strategies, and therefore did not follow the kinematic classification proposed by Di Marco et al. (2019) [7]. In that previous study, an 'atypical crouch' gait pattern was distinguished from a 'straight' pattern based on knee angle at initial contact. Their findings of anterior pelvic tilt, increased hip and knee flexion and external foot progression angles were confirmed in the present study. Furthermore, three main kinetic strategies were revealed that underly the observed deviations. These results imply that clinical evaluation of gait should pay attention to knee angles, trunk lean and $M_{S}$. When forward trunk leaning is observed, kinetic analysis can reveal whether this potential compensation for crouch gait was successful. 
Lever arm dysfunction and weakness or impaired control of muscles were identified as possible causes of crouch gait in patients with cerebral palsy [14]. External tibial torsion and pes planovalgus were frequently observed in patients with DS, decreasing the lever arm of the foot, which may disrupt the plantar flexion-knee extension couple. However, these malalignments we equally distributed over the three subgroups and might not impose the kinetic strategies. Measuring muscle strength was too challenging owing to cognitive impairments.

Age differences revealed that compensated crouch gait was mostly observed in younger children, which might evolve to uncompensated crouch in adolescence. These findings are in line with previous research suggesting progressive deterioration of gait [5] The progression of crouch gait as observed in cerebral palsy, involves the risk to develop hamstrings tightening with knee flexion contractures and loss of functional independency [30]. Functional mobility was indeed more limited in KMext. Hamstrings tightening was present in half of the participants, independent from their kinetic strategies, but flexion contractures at the knee joint were only observed in KMext (two patients). Longitudinal studies are needed to document the evolution and detect prognostic factors.

Other clinical characteristics did not differ significantly between subgroups. This may suggest that epileptic activity and cognitive development were not determinative for gait strategies. However, due to small numbers of participants per subgroup and heterogeneity within DS, associations might be hard to detect. Ideally, gait interventions prevent crouch gait development and help patients achieve an overall more efficient gait pattern with a favourable functional outcome. Further research on causal mechanisms behind mechanical deviations in DS could guide therapy.

The $M_{S}$ is limited to the sagittal plane, where major kinetic strategies were situated. However, coronal and transverse plane deviations were also observed. Internal hip abduction moments contribute to stance limb support and were characterised by a decreased second peak, which might be explained by step width or lateral trunk motion. Cross-plane interactions could be the subject of future research.

\section{Limitations}

A first limitation of this study were cognitive and behavioural problems that challenged participants to comply with rigorous protocols of gait analysis. This has led to the exclusion of four participants. The sample was large and therefore strongly representative for a rare disorder. Nevertheless, it remains uncertain whether the included patients' gait was representative for patients with the most severe cognitive and behavioural problems. A second limitation was the large age range of the included patients. Assembling childhood, adolescent and young adult clinical presentation may have increased the heterogeneity of the sample, but also offered an opportunity to demonstrate the diversity in gait deviations from early to adult age. This study accounted for effects of height and weight differences by non-dimensional normalisation of spatiotemporal parameters and joint moments [23]. Furthermore, the use of video images to assess trunk lean was less objective and reliable than quantitative registration using trunk markers, which would be recommended for future instrumented gait analysis in this population [31].

\section{Conclusion}

An overall inefficient walking pattern was evident in patients with DS. Increased $M_{S}$ suggested that more extensor muscle effort was required to maintain stance limb stability. Forward trunk lean was mostly employed by younger patients with various degrees of success to attain alignment of the ground reaction force that 
facilitates knee extension. Closer-to-normal kinetic strategies were as well observed, with mild gait deviations and a favourable functional outcome.

\section{Acknowledgements and conflict of interest statement}

The authors thank the participants and their families for their cooperation and prof. dr. Guy Molenaers for his advice. This study was supported by the Flemish Research Council (grant number T003116N), the University of Antwerp, the University Hospital of Antwerp and the KU Leuven. The authors declare that they have no conflict of interest. 


\section{References}

[1] C. Dravet, The core Dravet syndrome phenotype, Epilepsia. 52 (2011) 3-9. doi:10.1111/j.15281167.2011.02994.x.

[2] I.E. Scheffer, S.F. Berkovic, G. Capovilla, M.B. Connolly, J.A. French, L. Guilhoto, E. Hirsch, S. Jain, G.W. Mathern, S.L. Moshé, D.R. Nordli, E. Perucca, T. Tomson, ILAE classification of the epilepsies: Position paper of the ILAE Commission for Classification and Terminology, Epilepsia. 58 (2017) 512 521. doi:10.1111/epi.13709.

[3] L. Claes, J. Del-Favero, B. Ceulemans, L. Lagae, C. Van Broeckhoven, P. De Jonghe, De novo mutations in the sodium-channel gene SCN1A cause severe myoclonic epilepsy of infancy, Am. J. Hum. Genet. (2001). doi:10.1086/320609.

[4] W.A. Catterall, Dravet syndrome: a sodium channel interneuronopathy, Curr. Opin. Physiol. 2 (2018) 42-50. doi:10.1016/j.cophys.2017.12.007.

[5] J.M. Rodda, I.E. Scheffer, J.M. McMahon, S.F. Berkovic, K. Graham, Progressive gait deterioration in adolescents with Dravet syndrome, Arch. Neurol. 69 (2012) 873-878. doi:10.1001/archneurol.2011.3275.

[6] L. Wyers, P. Van de Walle, A. Hoornweg, I.T. Bobescu, K. Verheyen, B. Ceulemans, A.-S. Schoonjans, K. Desloovere, A. Hallemans, J.M. Rodda, I. Tepes Bobescu, Gait deviations in patients with dravet syndrome: A systematic review, Eur. J. Paediatr. Neurol. 23 (2019) 357-367. doi:10.1016/j.ejpn.2019.03.003.

[7] R. Di Marco, A. Hallemans, G. Bellon, F. Ragona, E. Piazza, T. Granata, B. Ceulemans, A.-S. Schoonjans, P. Van de Walle, F. Darra, B. Dalla Bernardina, M. Vecchi, Z. Sawacha, B. Scarpa, S. Masiero, M.G. Benedetti, A. Del Felice, Gait abnormalities in people with Dravet syndrome: A crosssectional multi-center study, Eur. J. Paediatr. Neurol. 23 (2019) 808-818. doi:10.1016/j.ejpn.2019.09.010.

[8] D. Sutherland, J.R. Davids, Common Gait Abnormalities of the Knee in Cerebral Palsy, Clin. Orthop. Relat. Res. 288 (1993) 139-147.

[9] J.M. Rodda, K. Graham, L. Carson, M.P. Galea, R. Wolfe, Sagittal gait patterns in spastic diplegia, J. Bone Jt. Surg. - Ser. B. 86 (2004) 251-258. doi:10.1302/0301-620X.86B2.13878.

[10] A. Brunklaus, R. Ellis, E. Reavey, G.H. Forbes, S.M. Zuberi, Prognostic, clinical and demographic features in SCN1A mutation-positive Dravet syndrome, Brain. 135 (2012) 2329-2336. doi:10.1093/brain/aws151.

[11] D.A. Winter, P. Eng, Kinetics: our window into the goals and strategies of the central nervous system, Behav. Brain Res. 67 (1995) 111-120. doi:10.1016/0166-4328(94)00154-8.

[12] D.A. Winter, Overall principle of lower limb support during stance phase of gait, J. Biomech. 13 (1980) 923-927.

[13] A.L. Hof, On the Interpretation of the Support Moment, Gait Posture. 12 (2000) 196-199. doi:10.1016/S0966-6362(00)00084-9.

[14] J.R. Gage, Gait Analysis; an essential tool in the treatment of Cerebral Palsy, Clin. Orthop. Relat. Res. 288 (1993) 126-134.

[15] L. Heyrman, H. Feys, G. Molenaers, E. Jaspers, D. Monari, A. Nieuwenhuys, K. Desloovere, Altered trunk movements during gait in children with spastic diplegia: Compensatory or underlying trunk control deficit?, Res. Dev. Disabil. 35 (2014) 2044-2052. doi:10.1016/j.ridd.2014.04.031.

[16] C. Gitiaux, N. Chemaly, S. Quijano-Roy, C. Barnerias, I. Desguerre, M. Hully, C. Chiron, O. Dulac, R. Nabbout, Motor neuropathy contributes to crouching in patients with Dravet syndrome, Neurology. 87 (2016) 277-281. doi:10.1212/WNL.0000000000002859. 
[17] D. Aljaafari, A. Fasano, F.A. Nascimento, A.E. Lang, D.M. Andrade, Adult motor phenotype differentiates Dravet syndrome from Lennox-Gastaut syndrome and links SCN1A to early onset parkinsonian features, Epilepsia. 58 (2017) e44-e48. doi:10.1111/epi.13692.

[18] R.B. Davis, S. Ounpuu, D. Tyburski, J.R. Gage, A gait analysis data collection and reduction technique, Hum. Mov. Sci. 10 (1991) 575-597.

[19] K. Graham, A. Harvey, J.M. Rodda, G.R. Nattrass, M. Pirpiris, The Functional Mobility Scale (FMS), J. Pediatr. Orthop. 24 (2004) 514-520. doi:10.1097/01241398-200409000-00011.

[20] S. Greenspan, G.W. Woods, Intellectual disability as a disorder of reasoning and judgement: The gradual move away from intelligence quotient-ceilings, Curr. Opin. Psychiatry. 27 (2014) 110-116. doi:10.1097/YCO.0000000000000037.

[21] A.J. Mudge, K. V. Bau, L.N. Purcell, J.C. Wu, M.W. Axt, P. Selber, J. Burns, Normative reference values for lower limb joint range, bone torsion, and alignment in children aged 4-16 years, J. Pediatr. Orthop. Part B. 23 (2014) 15-25. doi:10.1097/BPB.0b013e328364220a.

[22] A.C. Redmond, Y.Z. Crane, H.B. Menz, Normative values for the Foot Posture Index, J. Foot Ankle Res. 1 (2008) 1-9. doi:10.1186/1757-1146-1-6.

[23] A.L. Hof, Scaling gait data to body size, Gait Posture. 4 (1996) 222-223. doi:10.1016/09666362(95)01057-2.

[24] O. Pinzone, M.H. Schwartz, R.W. Baker, Comprehensive non-dimensional normalization of gait data, Gait Posture. 44 (2016) 68-73. doi:10.1016/j.gaitpost.2015.11.013.

[25] H.S. Read, M.E. Hazlewood, S.J. Hillman, R.J. Prescott, J.E. Robb, Edinburgh visual gait score for use in cerebral palsy, J. Pediatr. Orthop. 23 (2003) 296-301. doi:10.1097/00004694-200305000-00005.

[26] T.C. Pataky, M.A. Robinson, J. Vanrenterghem, Vector field statistical analysis of kinematic and force trajectories, J. Biomech. 46 (2013) 2394-2401. doi:10.1016/j.jbiomech.2013.07.031.

[27] T.C. Pataky, Generalized n-dimensional biomechanical field analysis using statistical parametric mapping, J. Biomech. 43 (2010) 1976-1982. doi:10.1016/j.jbiomech.2010.03.008.

[28] S. Lydersen, V. Pradhan, P. Senchaudhuri, P. Laake, Choice of test for association in small sample unordered r x c tables, 26 (2007) 4328-4343. doi:10.1002/sim.2839.

[29] J.R. Davids, A.M. Bagley, Identification of common gait disruption patterns in children with cerebral palsy, J. Am. Acad. Orthop. Surg. 22 (2014) 782-790. doi:10.5435/JAAOS-22-12-782.

[30] R. O'Sullivan, F. Horgan, T. O'Brien, H. French, The natural history of crouch gait in bilateral cerebral palsy: A systematic review, Res. Dev. Disabil. 80 (2018) 84-92. doi:10.1016/j.ridd.2018.06.013.

[31] J. Romkes, W. Peeters, A.M. Oosterom, S. Molenaar, I. Bakels, R. Brunner, Evaluating upper body movements during gait in healthy children and children with diplegic cerebral palsy, J. Pediatr. Orthop. Part B. 16 (2007) 175-180. doi:10.1097/BPB.0b013e32801405bf. 


\section{FIGURES AND TABLES}

\begin{tabular}{|c|c|c|c|}
\hline & $\operatorname{DS}(n=41)$ & TD $(n=41)$ & $P$-value \\
\hline & median (IQR) & median (IQR) & Wilcoxon \\
\hline \multicolumn{4}{|l|}{ Demographics: } \\
\hline Age (years) & $11.4(10.1)$ & $12.0(9.1)$ & 0.98 \\
\hline Gender (f / m) & $19 / 22$ & $27 / 14$ & \\
\hline BMI & $17.0(8.6)$ & $17.1(5.4)$ & 0.68 \\
\hline \multicolumn{4}{|l|}{ Spatiotemporal parameters: } \\
\hline Walking velocity $(\mathrm{m} / \mathrm{sec})$ & $1.03(0.37)$ & $1.21(0.20)$ & \\
\hline Non-dimensional walking velocity & $0.360(0.136)$ & $0.460(0.082)$ & $<0.001 *$ \\
\hline Cadence (steps/sec) & $2.01(0.45)$ & $2.02(0.33)$ & \\
\hline Non-dimensional cadence & $0.570(0.091)$ & $0.560(0.048)$ & 0.71 \\
\hline Step length (m) & $0.49(0.18)$ & $0.55(0.15)$ & \\
\hline Non-dimensional step length & $0.650(0.166)$ & $0.760(0.155)$ & $0.006 *$ \\
\hline Step width (m) & $0.16(0.06)$ & $0.10(0.04)$ & \\
\hline Non-dimensional step width & $0.220(0.096)$ & $0.130(0.061)$ & $<0.001 *$ \\
\hline Stance time (\%GC) & $60.4(4.4)$ & $59.3(2.5)$ & $0.02 *$ \\
\hline
\end{tabular}

Table 1: Comparison of patient and control group. Median and interquartile range (IQR) with $P$-value of the Wilcoxon rank-sum test. *Significant at $\alpha=0.05$; DS, Dravet Syndrome; TD, typically developing; f, female; m, male; BMI, Body Mass Index. 


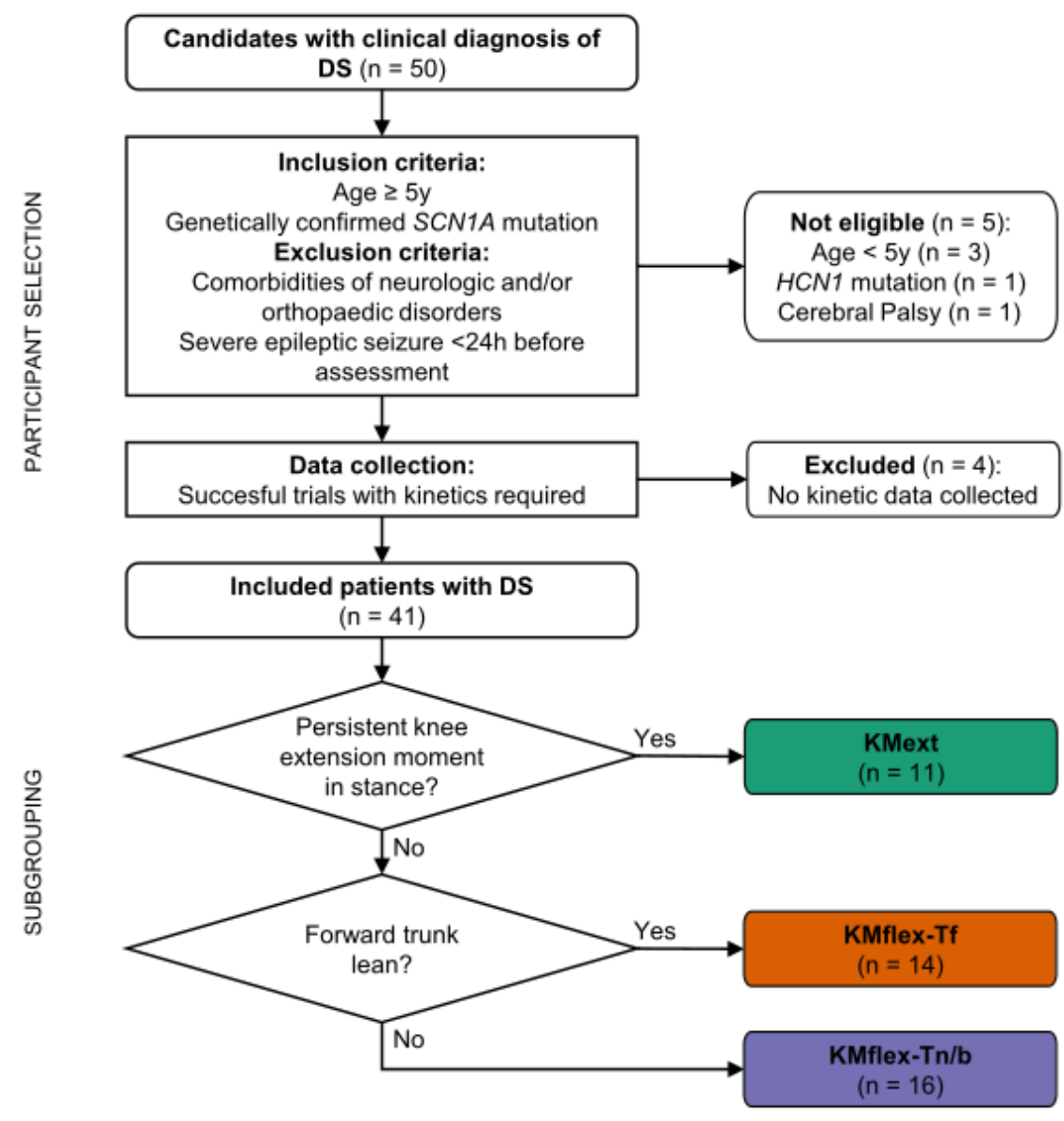

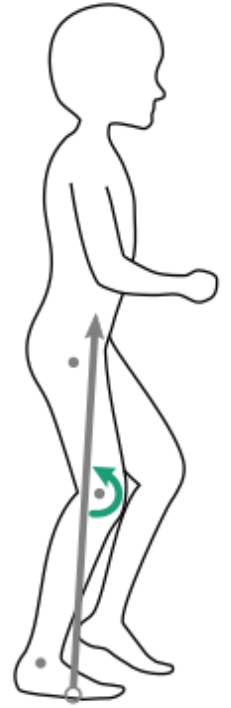

KMext

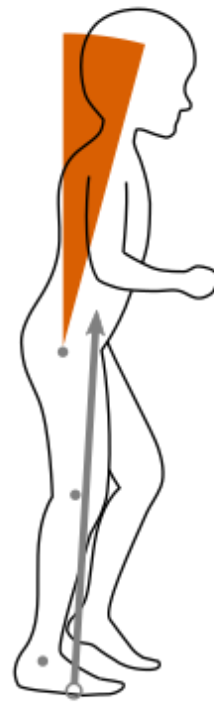

KMflex-Tf

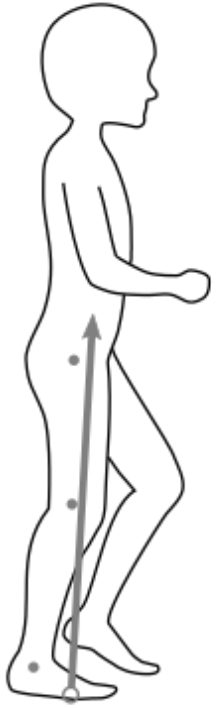

KMflex-Tn/b

Figure 1: Illustrated flow chart of the participant selection and subgrouping process. Patients who fail to align the GRF (grey arrow) in front of the knee, show a persistent internal knee extension moment (green arrow). Forward trunk lean $\left(>5^{\circ}\right.$; orange angle) influences the direction of the GRF. DS, Dravet Syndrome; TD, 
Typically Developing; KM, internal knee moment flexion (flex) or extension (ext); T, trunk lean forward (f) neutral (n) or backward (b). 

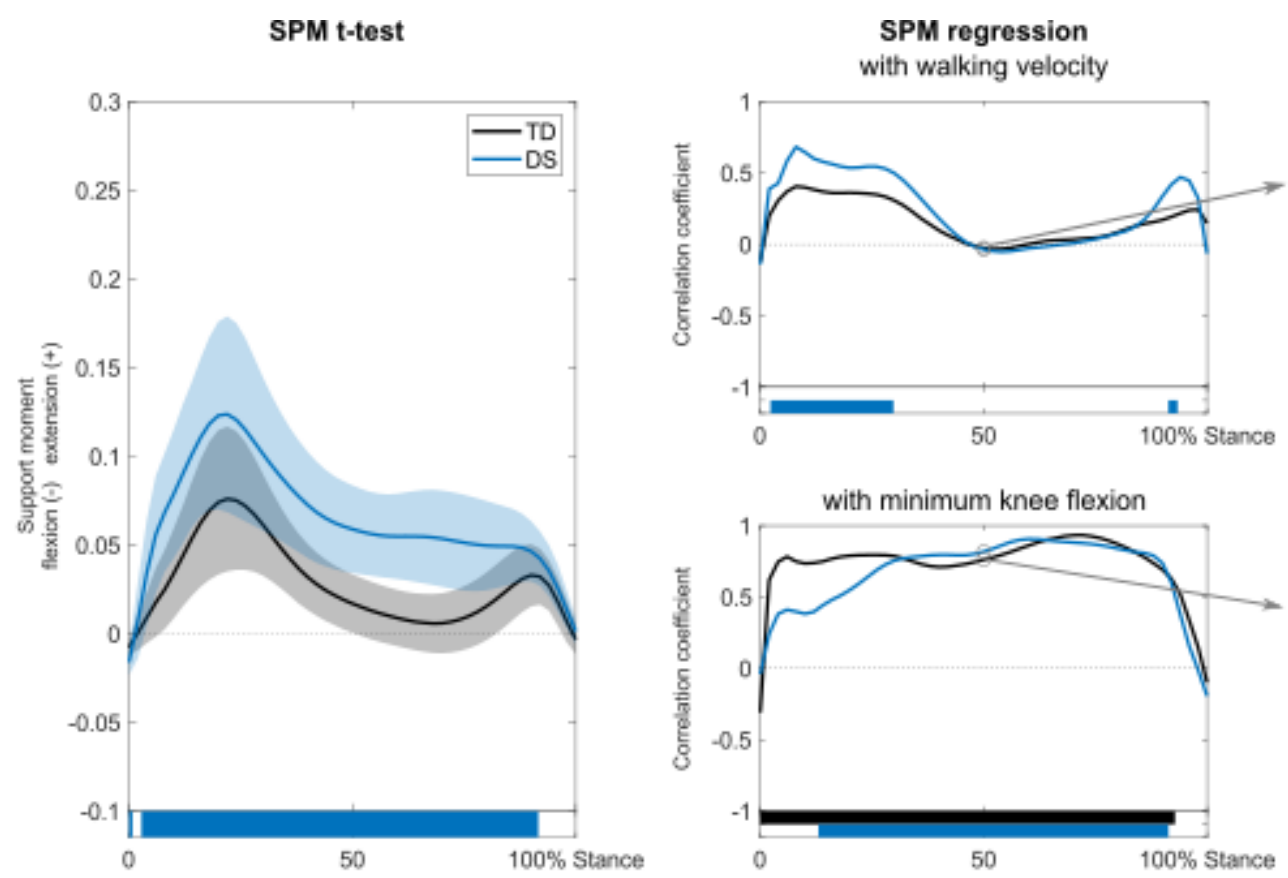

Correlation in midstance
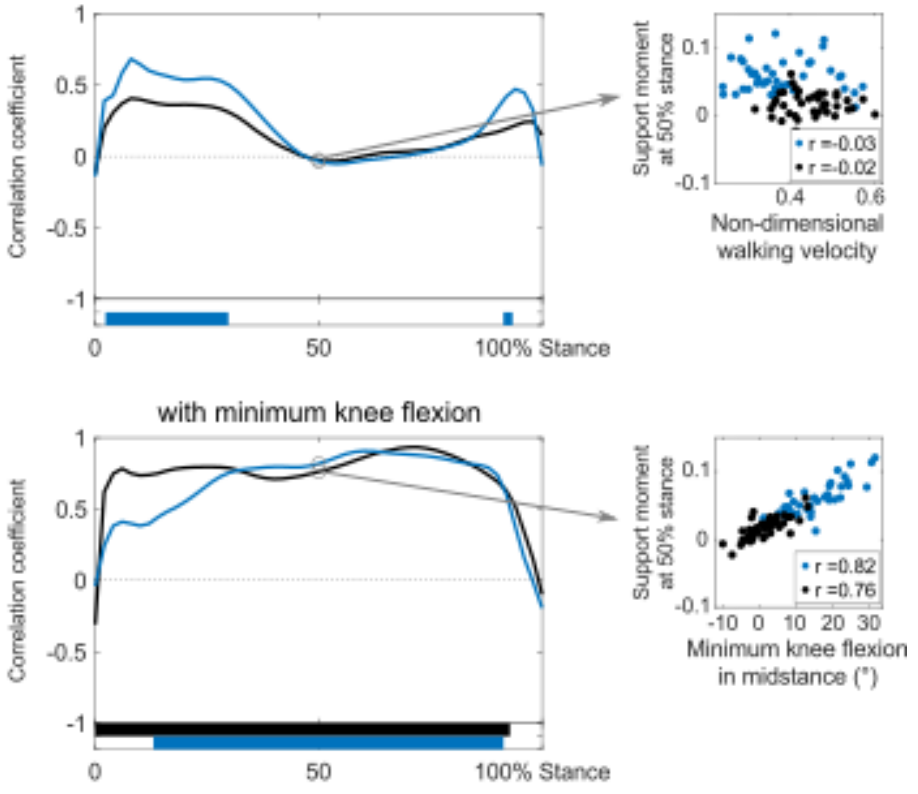

Figure 2: The support moment and its association with walking velocity and minimum knee flexion. Left: The non-dimensional support moment (averaged curve with 1SD region) was increased in patients with Dravet Syndrome (DS, blue) compared to typically developing participants (TD, black) Middle: Coefficients of the correlation between non-dimensional sagittal support moment and walking velocity (upper) and minimum knee flexion angle in midstance (lower). Right: In midstance, association with walking velocity was absent, but with minimum knee flexion was significant, as illustrated for $50 \%$ of stance phase. Horizontal bars represent clusters with significant differences (SPM $t$-test, left) or significant associations (SPM regression, middle). 

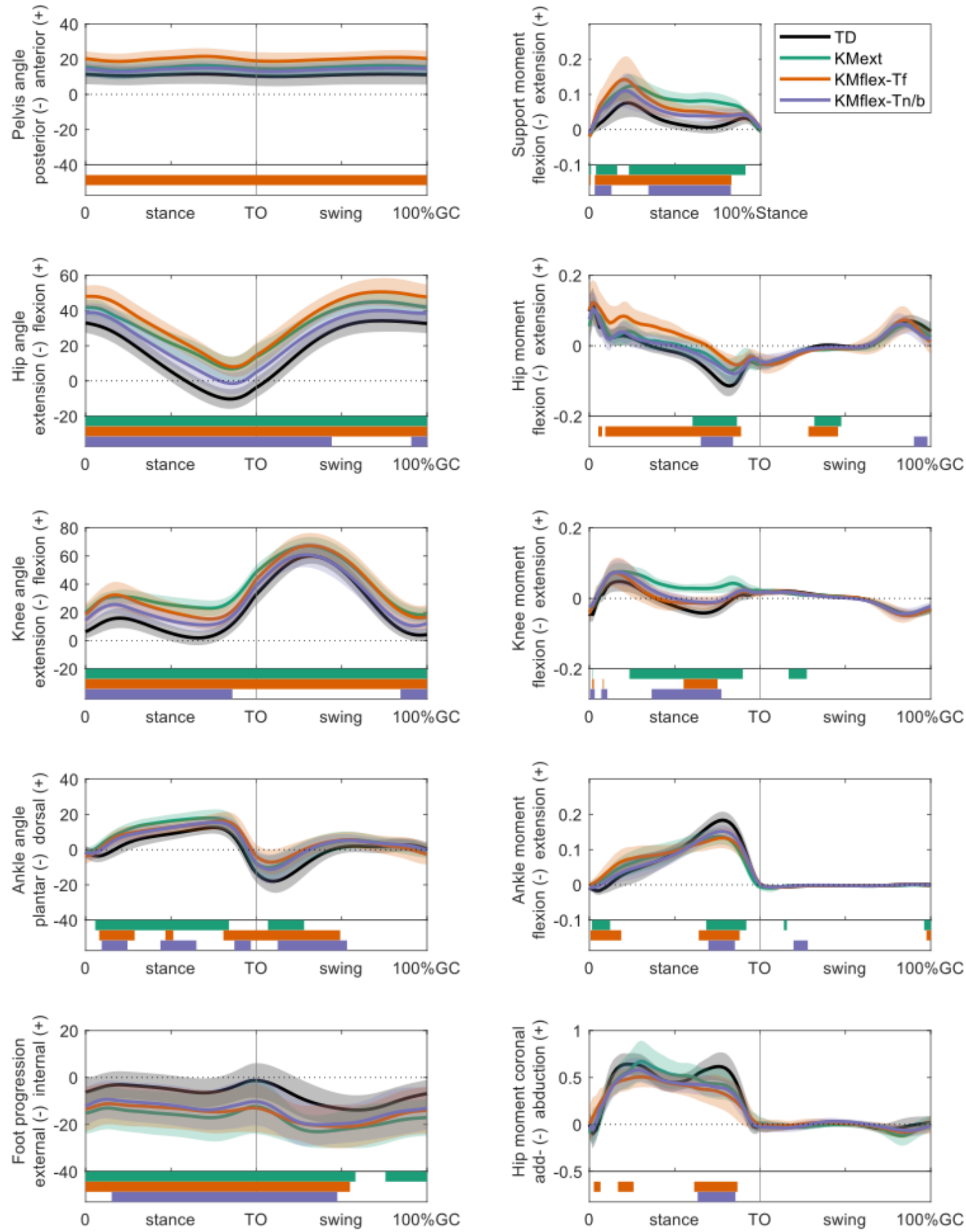

Figure 3: Comparison of kinematic and kinetic curves between Dravet Syndrome subgroups and typically developing (TD) controls. Group averaged curves and 1SD regions are plotted for joint angles $\left(^{\circ}\right)$ and internal net joint moments (non-dimensional) in the sagittal plane, complemented with the foot progression angle (under,

left) and hip moment in the coronal plane (under, right). Horizontal bars represent clusters with significant differences (SPM $t$-tests) comparing subgroups with the TD group. TO, toe off; GC, gait cycle. 


\begin{tabular}{|c|c|c|c|c|c|c|}
\hline & $\begin{array}{l}\text { KMext } \\
(\mathrm{n}=11)\end{array}$ & $\begin{array}{l}\text { KMflex-Tf } \\
(n=14)\end{array}$ & $\begin{array}{c}\text { KMflex-Tn/b } \\
(\mathbf{n}=16)\end{array}$ & \multicolumn{3}{|c|}{$P$-value } \\
\hline & $\begin{array}{l}\text { median (IQR) } \\
\text { or } \mathrm{n}(\%)\end{array}$ & $\begin{array}{l}\text { median (IQR) } \\
\text { or } \mathrm{n}(\%)\end{array}$ & $\begin{array}{l}\text { median (IQR) } \\
\text { or } \mathrm{n}(\%)\end{array}$ & $\mathrm{K}-\mathrm{W}$ & Fisher & Post-hoc \\
\hline Age (years) & $17.1(5.5)$ & $9.2(6.9)$ & $11.1(8.9)$ & 0.04 & & 0.03 (a) \\
\hline Gender (f / m) & $5 / 6$ & $7 / 7$ & $7 / 9$ & & & \\
\hline BMI & $19.2(11.0)$ & $17.1(4.5)$ & $16.1(5.7)$ & 0.45 & & \\
\hline Age of onset epilepsy (months) & $5(4)$ & $5(4)$ & $5(2)$ & 0.80 & & \\
\hline Current epileptic frequency & & & & & 0.17 & \\
\hline Free (for $>1$ year) & $1(9 \%)$ & $3(21 \%)$ & $6(38 \%)$ & & & \\
\hline Mild (yearly) & $5(45 \%)$ & $5(43 \%)$ & $4(25 \%)$ & & & \\
\hline Moderate (monthly) & $0(0 \%)$ & $3(21 \%)$ & $4(25 \%)$ & & & \\
\hline Severe (weekly) & $5(45 \%)$ & $2(14 \%)$ & $2(13 \%)$ & & & \\
\hline Intellectual disability: & & & & & 0.42 & \\
\hline Mild & $3(27 \%)$ & $2(14 \%)$ & $6(38 \%)$ & & & \\
\hline Moderate & $3(27 \%)$ & $6(43 \%)$ & $7(44 \%)$ & & & \\
\hline Severe & $5(45 \%)$ & $6(43 \%)$ & $3(19 \%)$ & & & \\
\hline $\begin{array}{l}\text { Age of independent walking } \\
\text { (months) }\end{array}$ & $18(10)$ & $18(1)$ & $16(4)$ & 0.18 & & \\
\hline Walking velocity (m/sec) & $1.08(0.28)$ & $1.04(0.44)$ & $1.00(0.31)$ & & & \\
\hline $\begin{array}{l}\text { Non-dimensional walking } \\
\text { velocity }\end{array}$ & $0.366(0.106)$ & $0.382(0.159)$ & $0.358(0.115)$ & 0.77 & & \\
\hline Walking distance & & & & & 0.02 & $\begin{array}{l}\mathbf{0 . 0 3}^{(\mathbf{b})} \\
\mathbf{0 . 0 0 9}^{(\mathrm{c})}\end{array}$ \\
\hline$<1 \mathrm{~km}$ & $5(45 \%)$ & $8(57 \%)$ & $1(7 \%)$ & & & \\
\hline $1 \mathrm{~km}-3 \mathrm{~km}$ & $3(27 \%)$ & $2(14 \%)$ & $3(20 \%)$ & & & \\
\hline$>3 \mathrm{~km}$ & $3(27 \%)$ & $4(29 \%)$ & $11(73 \%)$ & & & \\
\hline FMS-5m: & & & & & & \\
\hline Score 4 & $0(0 \%)$ & $1(7 \%)$ & $0(0 \%)$ & & & \\
\hline Score 5 & $10(91 \%)$ & $11(79 \%)$ & $10(67 \%)$ & & & \\
\hline Score 6 & $1(9 \%)$ & $2(14 \%)$ & $5(33 \%)$ & & & \\
\hline FMS-50m: & & & & & & \\
\hline Score 4 & $3(27 \%)$ & $6(43 \%)$ & $1(7 \%)$ & & & \\
\hline Score 5 & $7(64 \%)$ & $6(43 \%)$ & $10(67 \%)$ & & & \\
\hline Score 6 & $1(9 \%)$ & $2(14 \%)$ & $4(27 \%)$ & & & \\
\hline FMS-500m: & & & & & 0.10 & $0.04^{(\mathrm{c})}$ \\
\hline Score 1 & $5(45 \%)$ & $8(57 \%)$ & $2(2 \%)$ & & & \\
\hline Score 4 & $3(27 \%)$ & $1(7 \%)$ & $3(23 \%)$ & & & \\
\hline Score 5 & $2(18 \%)$ & $5(36 \%)$ & $7(47 \%)$ & & & \\
\hline Score 6 & $1(9 \%)$ & $0(0 \%)$ & $3(20 \%)$ & & & \\
\hline
\end{tabular}

Table 2: Clinical characteristics of the three subgroups. Median and interquartile range (IQR) with $P$-value of the Kruskal-Wallis test (K-W) for numerical data. Count and percentage (\%) with $P$-value of the Fisher's exact test (Fisher) for categorical data. Significantly different values are in bold, with $P \leq 0.05$ for KruskalWallis and $P \leq 0.10$ for Fisher's exact test. $P$-values of post hoc tests were only reported when significant with ${ }^{(a)}$ 
between KMext and KMflex-Tf, ${ }^{(b)}$ between KMext and KMflex-Tn/b, and ${ }^{(c)}$ between KMflex-Tf and KMflex$\mathrm{Tn} / \mathrm{b}$. FMS: Functional Mobility Scale. FMS values were missing for one participant in the KMflex-Tn/b group. 


\begin{tabular}{|c|c|c|c|}
\hline \multirow[t]{2}{*}{ Test (method) } & $\begin{array}{l}\text { KMext } \\
(\mathrm{n}=11)\end{array}$ & $\begin{array}{l}\text { KMflex-Tf } \\
(n=14)\end{array}$ & $\begin{array}{l}\text { KMflex-Tn/b } \\
\quad(n=16)\end{array}$ \\
\hline & $\begin{array}{l}\text { median }(\mathrm{IQR}) \\
\text { or } \mathrm{n}(\%)\end{array}$ & $\begin{array}{l}\text { median (IQR) } \\
\text { or } \mathrm{n}(\%)\end{array}$ & $\begin{array}{l}\text { median }(\mathrm{IQR}) \\
\text { or } \mathrm{n}(\%) \\
\end{array}$ \\
\hline \multicolumn{4}{|l|}{ Joint ROM and muscle length } \\
\hline \multicolumn{4}{|l|}{ Hip extension, $\mathrm{m}$ psoas (Thomas test) } \\
\hline Normal & $7(64 \%)$ & $12(86 \%)$ & $12(75 \%)$ \\
\hline Limited & $4(36 \%)$ & $2(14 \%)$ & $4(25 \%)$ \\
\hline \multicolumn{4}{|l|}{ Knee flexion, m rectus femoris (Duncan Ely) } \\
\hline Normal & $3(27 \%)$ & $10(71 \%)$ & $8(50 \%)$ \\
\hline Limited & $8(73 \%)$ & $4(29 \%)$ & $8(50 \%)$ \\
\hline \multicolumn{4}{|l|}{$\begin{array}{l}\text { Knee extension, joint ROM (positive values for } \\
\text { hyperextension) }\end{array}$} \\
\hline Median (IQR) & $0(7.5)$ & $5(10)$ & $5(6.25)$ \\
\hline Normal & $9(82 \%)$ & $14(100 \%)$ & $16(100 \%)$ \\
\hline Limited & $2(18 \%)$ & $0(0 \%)$ & $0(0 \%)$ \\
\hline \multicolumn{4}{|l|}{$\begin{array}{l}\text { Knee extension, m hamstrings (Popliteal angle } \\
\text { with contralateral knee extended) }\end{array}$} \\
\hline Median (IQR) & $-45(12.5)$ & $-27.5(27.5)$ & $-37.5(21.25)$ \\
\hline Normal & $3(27 \%)$ & $9(64 \%)$ & $8(50 \%)$ \\
\hline Limited & $8(73 \%)$ & $5(36 \%)$ & $8(50 \%)$ \\
\hline \multicolumn{4}{|l|}{ Ankle dorsiflexion, m soleus (Silfverskiöld $90^{\circ}$ ) } \\
\hline Median (IQR) & $10(10)$ & $20(8.75)$ & $17.5(10)$ \\
\hline Normal & $1(9 \%)$ & $3(21 \%)$ & $4(25 \%)$ \\
\hline Limited & $10(91 \%)$ & $11(79 \%)$ & $12(75 \%)$ \\
\hline \multicolumn{4}{|l|}{$\begin{array}{l}\text { Ankle dorsiflexion, } \mathrm{m} \text { gastrocnemius } \\
\text { (Silfverskiöld } 0^{\circ} \text { ) }\end{array}$} \\
\hline Median (IQR) & $5(5)$ & $10(8.75)$ & $10(10)$ \\
\hline Normal & $1(9 \%)$ & $2(14 \%)$ & $4(25 \%)$ \\
\hline Limited & $10(91 \%)$ & $12(86 \%)$ & $12(75 \%)$ \\
\hline \multicolumn{4}{|l|}{ Skeletal alignment } \\
\hline \multicolumn{4}{|l|}{ Femoral anteversion (TPAT) } \\
\hline Median (IQR) & $25(5)$ & $30(5)$ & $30(5)$ \\
\hline Normal & $8(73 \%)$ & $11(79 \%)$ & $14(88 \%)$ \\
\hline Increased anteversion & $3(27 \%)$ & $3(21 \%)$ & $2(13 \%)$ \\
\hline \multicolumn{4}{|l|}{ Tibial torsion (bimalleolar angle) } \\
\hline Median (IQR) & $25(7.5)$ & $30(5)$ & $25(10)$ \\
\hline Normal & $3(27 \%)$ & $3(21 \%)$ & $6(38 \%)$ \\
\hline Increased external torsion & $8(73 \%)$ & $11(79 \%)$ & $10(63 \%)$ \\
\hline \multicolumn{4}{|l|}{ Foot posture index (FPI-6) } \\
\hline Median (IQR) & $6(5.5)$ & $5.5(6.75)$ & $6.5(6)$ \\
\hline Normal & $5(45 \%)$ & $9(64 \%)$ & $10(63 \%)$ \\
\hline Increased pronation (planovalgus) & $6(55 \%)$ & $4(29 \%)$ & $5(31 \%)$ \\
\hline Increased supination (cavovarus) & $0(0 \%)$ & $1(7 \%)$ & $1(6 \%)$ \\
\hline
\end{tabular}

Table 3: Physical examination of DS subgroups. All measures were compared to age-related norm values with mean \pm 1 SD as cut-off to define deviations (Mudge et al. 2014; Redmond et al. 2008). Values in this table did not significantly differ between subgroups. IQR, interquartile range; TPAT, trochanteric prominence angle test; ROM range of motion. 


\section{APPENDIX}
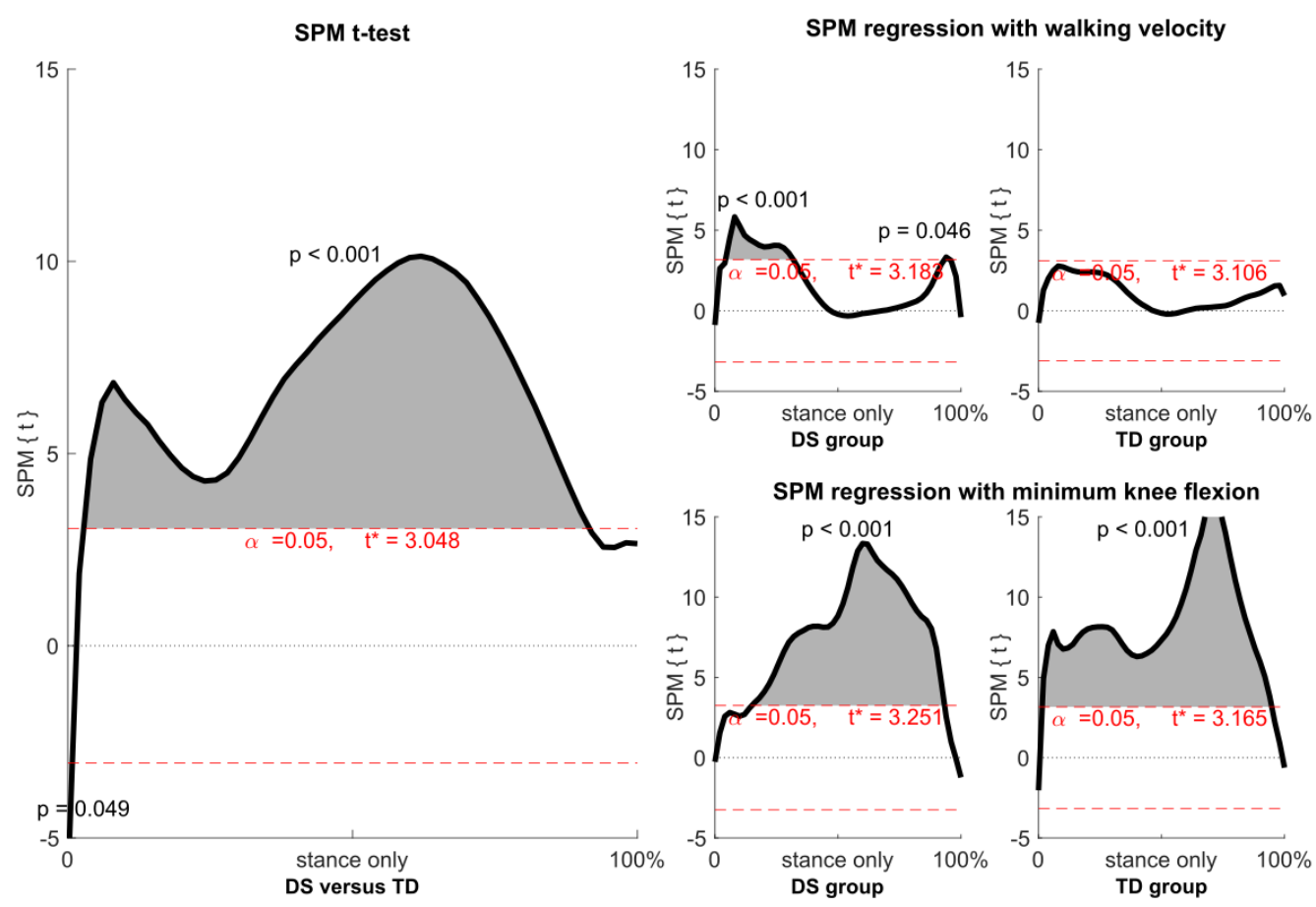

Figure A1: SPM inference curves accompanying figure 2: The support moment and its relation to walking velocity and knee flexion. Left: The $\operatorname{SPM}\{\mathrm{t}\}$ curve of the $t$ test statistics represents the difference between the support moment in the typically developing (TD) group and the Dravet Syndrome (DS) group over the stance phase. Right: The SPM $\{t\}$ curve expresses the effect magnitude of non-dimensional walking velocity (upper) and minimum knee flexion angle in midstance (lower) on the support moment in both groups. Red dashed lines indicate the critical treshold ( $\mathrm{t}^{*}$ ) for $\alpha=0.05$. Shaded areas with $P$ values are clusters with significant differences $(t$-test) or association (regression). 

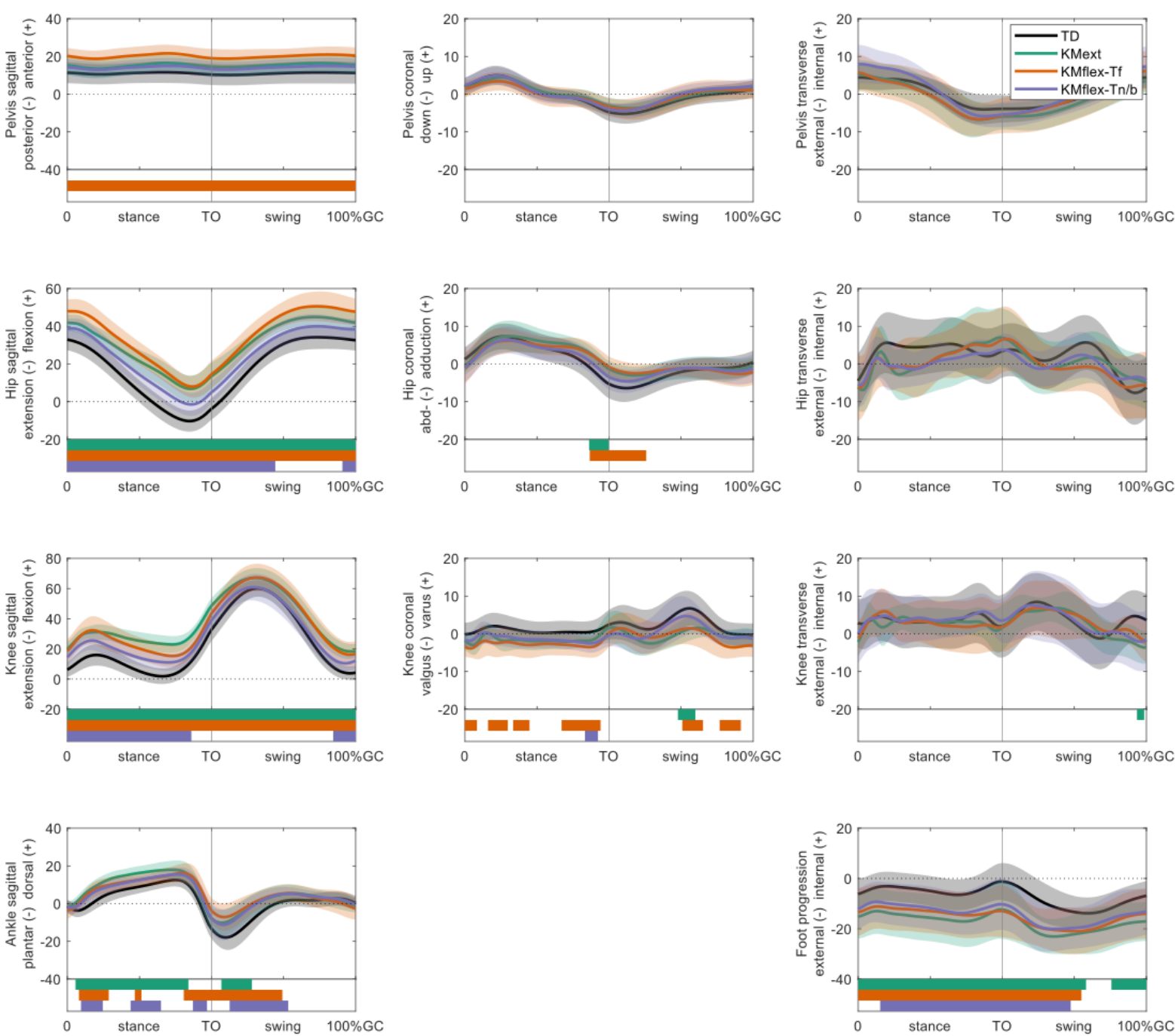

Figure A2: Comparison of kinematics in the three planes between Dravet Syndrome subgroups and typically developing (TD) controls. Group averaged curves and 1SD regions are plotted. Horizontal bars represent clusters with significant differences (SPM $t$-tests) comparing subgroups with the TD group. TO, toe off; GC, gait cycle. 
Kinematics (joint angles)
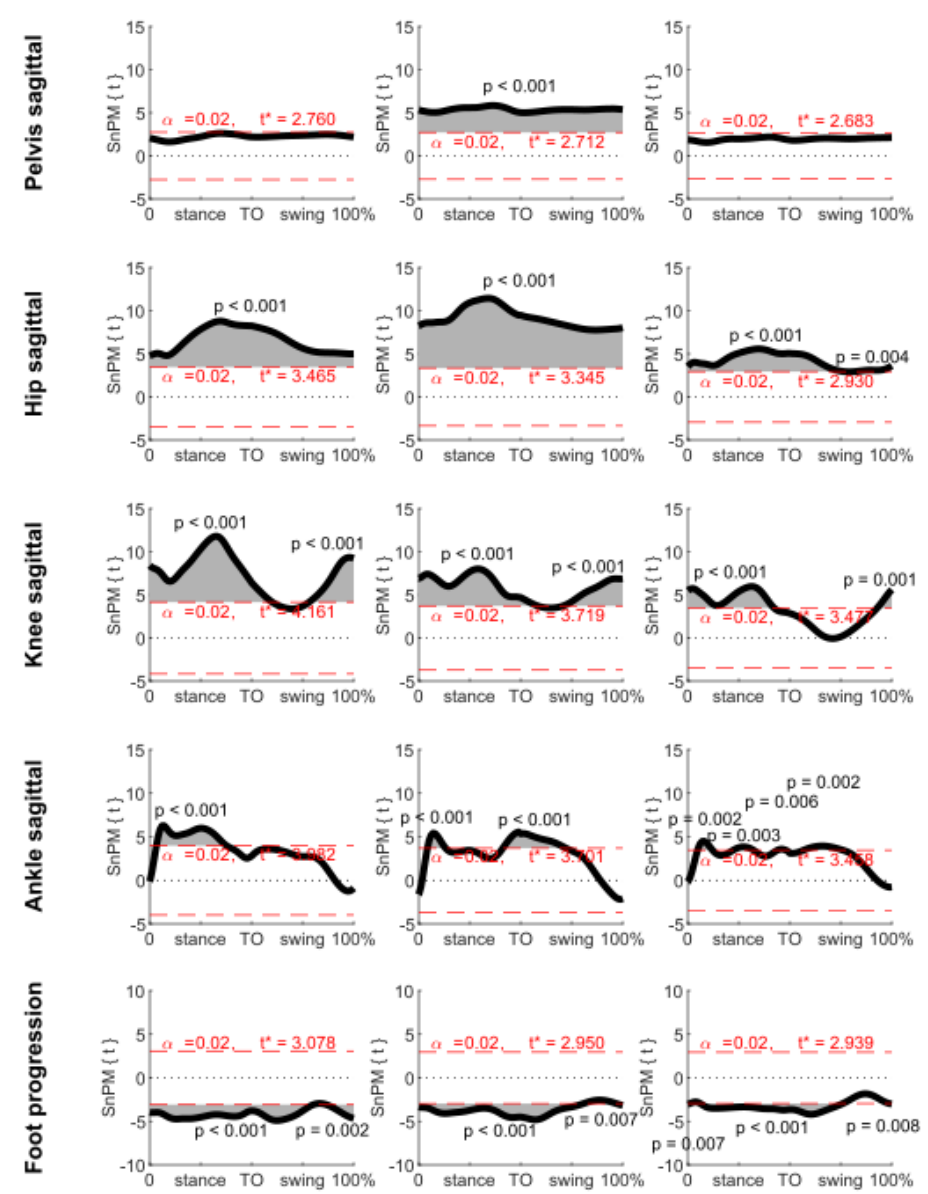

KMext

KMflex-Tf

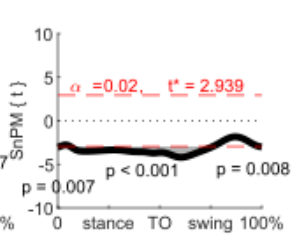

KMflex-Tn/b
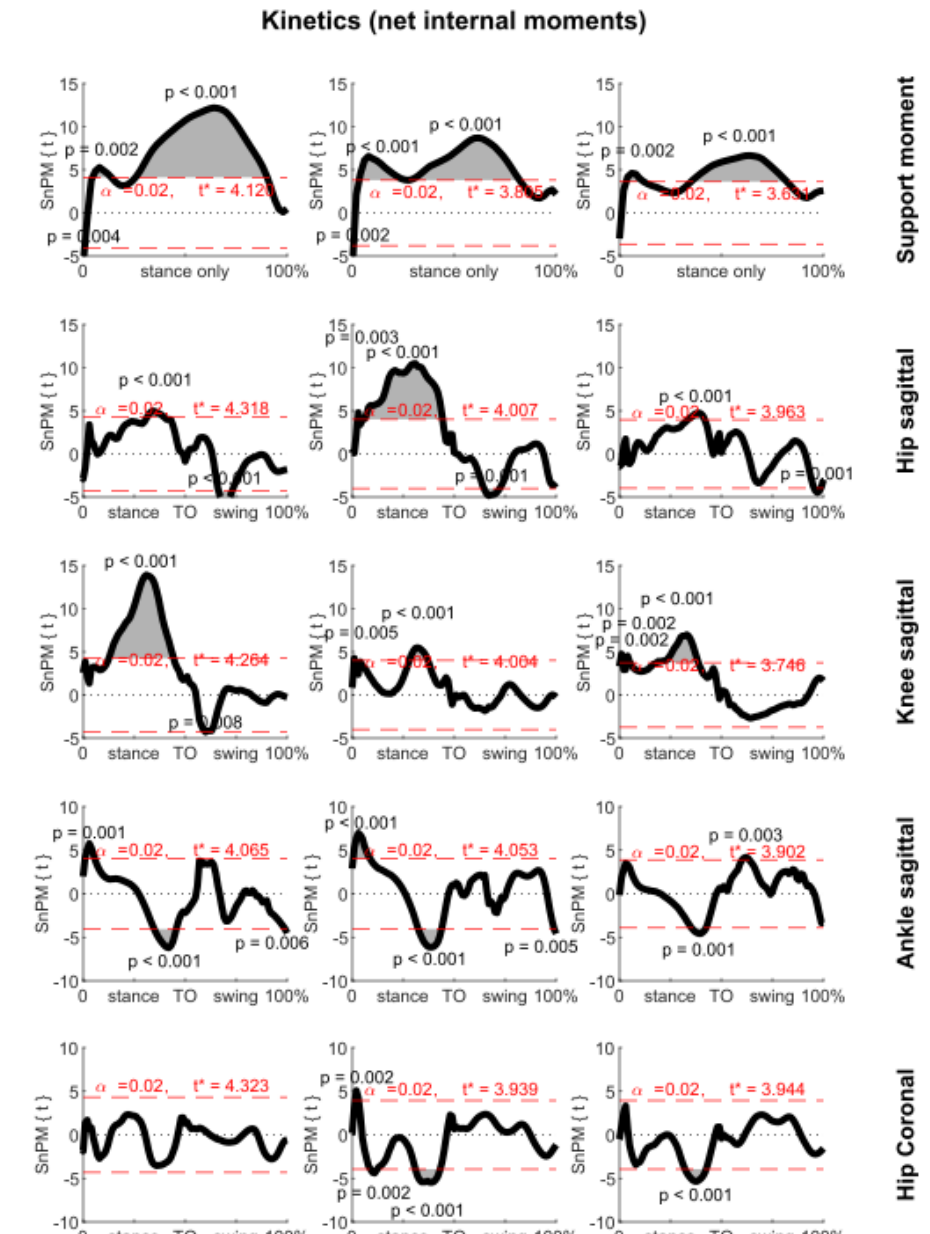

KMext

KMflex-Tf

KMflex-Tn/b

Figure A3: SPM inference curves accompanying figure 3: Comparison of kinematic and kinetic curves between Dravet Syndrome (DS) subgroups and typically developing (TD) controls. The SPM $\{\mathrm{t}\}$ curves of the $t$ test statistics represent the difference between the DS subgroup (KMext, KMflex-Tf or KMflex-Tn/b) and the TD group. Red dashed lines indicate the critical treshold ( $\mathrm{t}^{*}$ ) for Bonferroni corrected $\alpha=0.05 / 3$. Shaded areas with $P$ values are clusters with significant differences. The gait cycle on the X-axis was normalized for stance (50\%) and swing (50\%) phase, except for the support moment, where only stance phase (100\%) was analyzed. TO, toe-off 


\begin{tabular}{|c|c|c|c|c|c|c|c|c|c|c|c|}
\hline & $\begin{array}{l}\text { Age (years) } \\
\text { and gender }\end{array}$ & BMI & $\begin{array}{c}S C N 1 A \\
\text { mutation type }\end{array}$ & Nucleotide change & De novo & $\begin{array}{c}\text { Age of onset } \\
\text { epilepsy } \\
\text { (months) }\end{array}$ & $\begin{array}{c}\text { Current } \\
\text { epileptic } \\
\text { activity }\end{array}$ & Level of ID & $\begin{array}{c}\text { Age of } \\
\text { independent } \\
\text { walking (months) }\end{array}$ & $\begin{array}{l}\text { Walking } \\
\text { distance }\end{array}$ & FMS \\
\hline \multirow{11}{*}{ 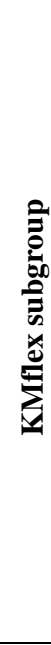 } & $6.4 \mathrm{~m}$ & 14.3 & missense & c. $3714 \mathrm{~A}>\mathrm{C}$ & no & 11 & mild & mild & 20 & $1 \mathrm{~km}-3 \mathrm{~km}$ & $5 / 5 / 1$ \\
\hline & $7.4 \mathrm{~m}$ & 14.7 & frameshift & c. $5536 \_5539 \mathrm{del}$ & yes & 4 & mild & mild & 18 & $1 \mathrm{~km}-3 \mathrm{~km}$ & $6 / 6 / 6$ \\
\hline & $13.4 \mathrm{f}$ & 17.9 & nonsense & c. $4219 \mathrm{C}>\mathrm{T}$ & unknown & 8 & severe & moderate & 13 & $1 \mathrm{~km}-3 \mathrm{~km}$ & $5 / 5 / 5$ \\
\hline & $16.1 \mathrm{f}$ & 27.0 & missense & c. $4294 \mathrm{~A}>\mathrm{G}$ & yes & 3 & mild & moderate & 30 & $<1 \mathrm{~km}$ & $5 / 5 / 1$ \\
\hline & $17.1 \mathrm{f}$ & 29.3 & missense & c. $4168 \mathrm{G}>\mathrm{A}$ & yes & 4 & free & mild & 14 & $>3 \mathrm{~km}$ & $5 / 5 / 5$ \\
\hline & $17.1 \mathrm{~m}$ & 14.3 & missense & c. $4223 \mathrm{G}>\mathrm{T}$ & yes & 8 & mild & moderate & 13 & $>3 \mathrm{~km}$ & $5 / 5 / 4$ \\
\hline & $19.2 \mathrm{f}$ & 23.7 & deletion & c.1200_1202delTGA & yes & 3 & mild & severe & 46 & $<1 \mathrm{~km}$ & $5 / 4 / 1$ \\
\hline & $19.8 \mathrm{~m}$ & 24.0 & missense & c. $680 \mathrm{~T}>\mathrm{G}$ & unknown & 3 & severe & severe & 27 & $<1 \mathrm{~km}$ & $5 / 5 / 1$ \\
\hline & $20.7 \mathrm{~m}$ & 14.4 & frameshift & c. $4497 \mathrm{delT}$ & yes & 6 & severe & severe & 18 & $<1 \mathrm{~km}$ & $5 / 4 / 1$ \\
\hline & $24.8 \mathrm{~m}$ & 19.2 & splice site & c. $4338+1 \mathrm{G}>\mathrm{A}$ & yes & 5 & severe & severe & 13 & $>3 \mathrm{~km}$ & $5 / 5 / 4$ \\
\hline & $25.7 \mathrm{f}$ & 28.2 & missense & c. $1178 \mathrm{G}>\mathrm{A}$ & yes & 6 & severe & severe & 18 & $<1 \mathrm{~km}$ & $5 / 4 / 4$ \\
\hline \multirow{11}{*}{ 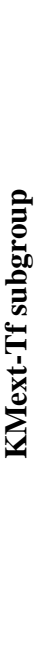 } & $5.2 \mathrm{~m}$ & 15.1 & missense & c. $5735 \mathrm{G}>\mathrm{C}$ & yes & 5 & mild & moderate & 18 & $1 \mathrm{~km}-3 \mathrm{~km}$ & $4 / 4 / 1$ \\
\hline & $5.2 \mathrm{f}$ & 17.2 & missense & c. $2836 \mathrm{C}>\mathrm{T}$ & unknown & 4 & mild & moderate & 37 & $<1 \mathrm{~km}$ & $5 / 5 / 1$ \\
\hline & $5.6 \mathrm{~m}$ & 16.3 & missense & c. $4346 \mathrm{~T}>\mathrm{C}$ & yes & 6 & mild & moderate & 18 & $<1 \mathrm{~km}$ & $5 / 4 / 1$ \\
\hline & $6.7 \mathrm{~m}$ & 13.7 & nonsense & c. $1348 \mathrm{C}>\mathrm{T}$ & yes & 8.5 & severe & moderate & 18 & $>3 \mathrm{~km}$ & $5 / 5 / 5$ \\
\hline & $7.2 \mathrm{f}$ & 19.7 & $\begin{array}{c}\text { intragenic } \\
\text { deletion }\end{array}$ & (exon 1-7) & unknown & 5 & severe & severe & 14 & $<1 \mathrm{~km}$ & $6 / 6 / 4$ \\
\hline & $7.5 \mathrm{~m}$ & 13.2 & missense & c. $406 \mathrm{~T}>\mathrm{C}$ & yes & 6 & free & mild & 18 & $>3 \mathrm{~km}$ & $5 / 5 / 5$ \\
\hline & $8.4 \mathrm{f}$ & 19.8 & missense & c. $1178 \mathrm{G}>\mathrm{A}$ & yes & 4 & free & severe & 18 & $<1 \mathrm{~km}$ & $5 / 5 / 1$ \\
\hline & $10.1 \mathrm{f}$ & 15.6 & missense & c. $296 \mathrm{~T}>\mathrm{A}$ & yes & 4 & mild & severe & 36 & $<1 \mathrm{~km}$ & $5 / 4 / 1$ \\
\hline & $10.6 \mathrm{f}$ & 18.0 & nonsense & c. $1738 \mathrm{C}>\mathrm{T}$ & yes & 5 & mild & severe & 18 & $<1 \mathrm{~km}$ & $5 / 4 / 1$ \\
\hline & $10.8 \mathrm{~m}$ & 13.4 & nonsense & c. $969 \mathrm{~T}>\mathrm{G}$ & yes & 3 & free & mild & 19 & $>3 \mathrm{~km}$ & $5 / 5 / 5$ \\
\hline & $14.7 \mathrm{f}$ & 23.2 & missense & c. $4633 A>G$ & yes & 11 & moderate & severe & unknown & $1 \mathrm{~km}-3 \mathrm{~km}$ & $6 / 6 / 5$ \\
\hline
\end{tabular}




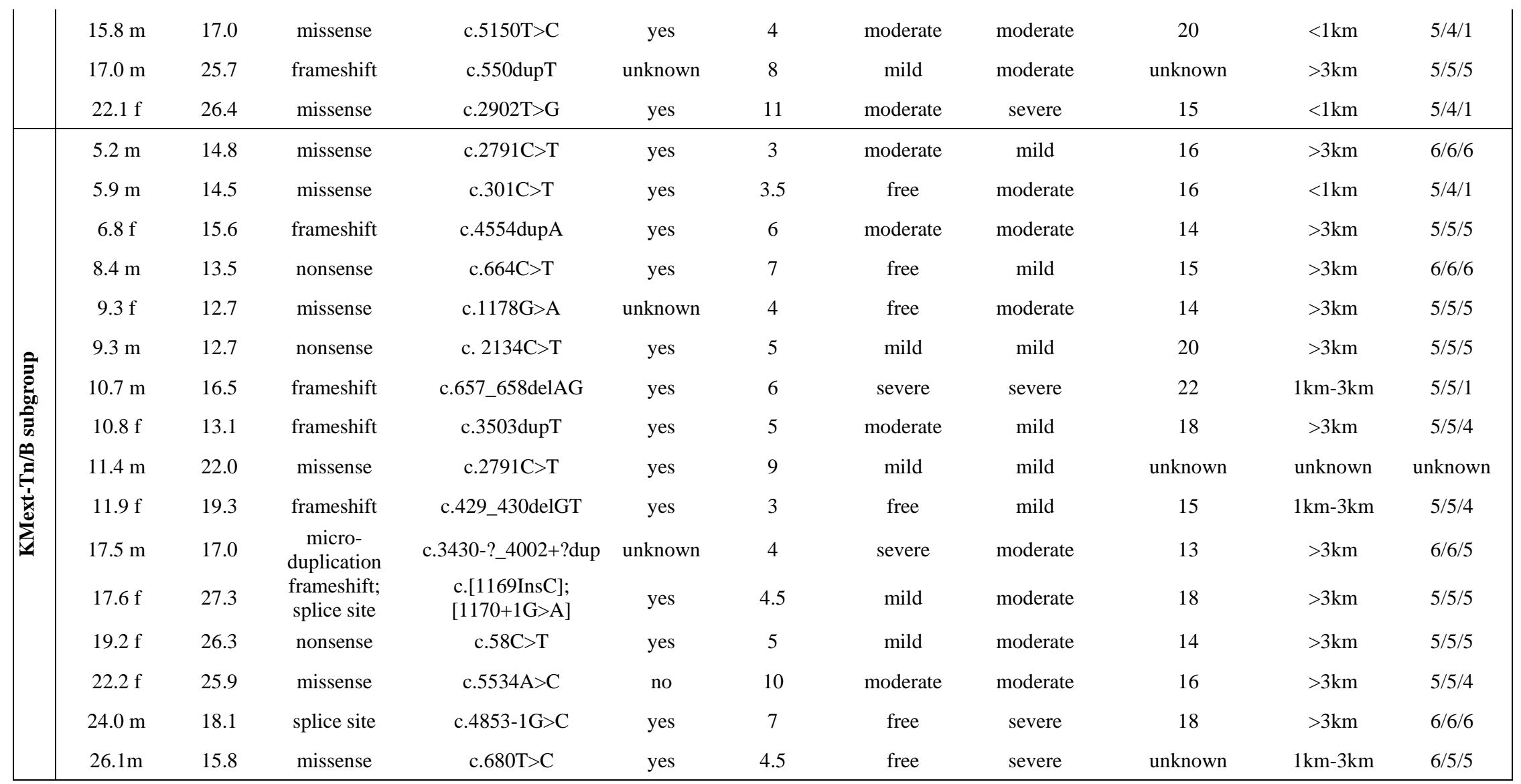

Table A1: Characteristics of individuals in the DS group. BMI = Body Mass Index, ID = Intellectual Disability, FMS = Functional Mobility Scale $(5 \mathrm{~m} / 50 \mathrm{~m} / 500 \mathrm{~m}), \mathrm{m}=$ male, $\mathrm{f}=$ female 


\begin{tabular}{|l|c|c|c|}
\hline & $\begin{array}{c}\text { KMext } \\
(\mathbf{n}=\mathbf{1 1})\end{array}$ & $\begin{array}{c}\text { KMflex-Tf } \\
(\mathbf{n}=\mathbf{1 4})\end{array}$ & $\begin{array}{c}\text { KMflex-Tn/b } \\
(\mathbf{n}=\mathbf{1 6})\end{array}$ \\
\cline { 2 - 4 } & median (IQR) & median (IQR) & median (IQR) \\
\hline Spatiotemporal parameters & & & \\
Walking velocity (m/sec) & $1.08(0.28)$ & $1.04(0.44)$ & $1.00(0.31)$ \\
Non-dimensional walking velocity & $0.366(0.106)$ & $0.382(0.159)$ & $0.358(0.115)$ \\
Cadence (steps/sec) & $1.92(0.27)$ & $2.08(0.53)$ & $2.02(0.56)$ \\
Non-dimensional cadence & $0.600(0.083)$ & $0.599(0.091)$ & $0.562(0.084)$ \\
Step length (m) & $0.51(0.17)$ & $0.46(0.11)$ & $0.52(0.14)$ \\
Non-dimensional step length & $0.595(0.156)$ & $0.637(0.213)$ & $0.659(0.101)$ \\
Step width (m) & $0.21(0.09)$ & $0.17(0.06)$ & $0.16(0.03)$ \\
Non-dimensional step width & $0.221(0.100)$ & $0.249(0.107)$ & $0.203(0.056)$ \\
Stance time (\%GC) & $61.7(3.2)$ & $60.9(5.7)$ & $59.6(3.6)$ \\
\hline
\end{tabular}

Table A2: Spatiotemporal parameters did not significantly differ between subgroups. IQR, interquartile range; GC, gait cycle
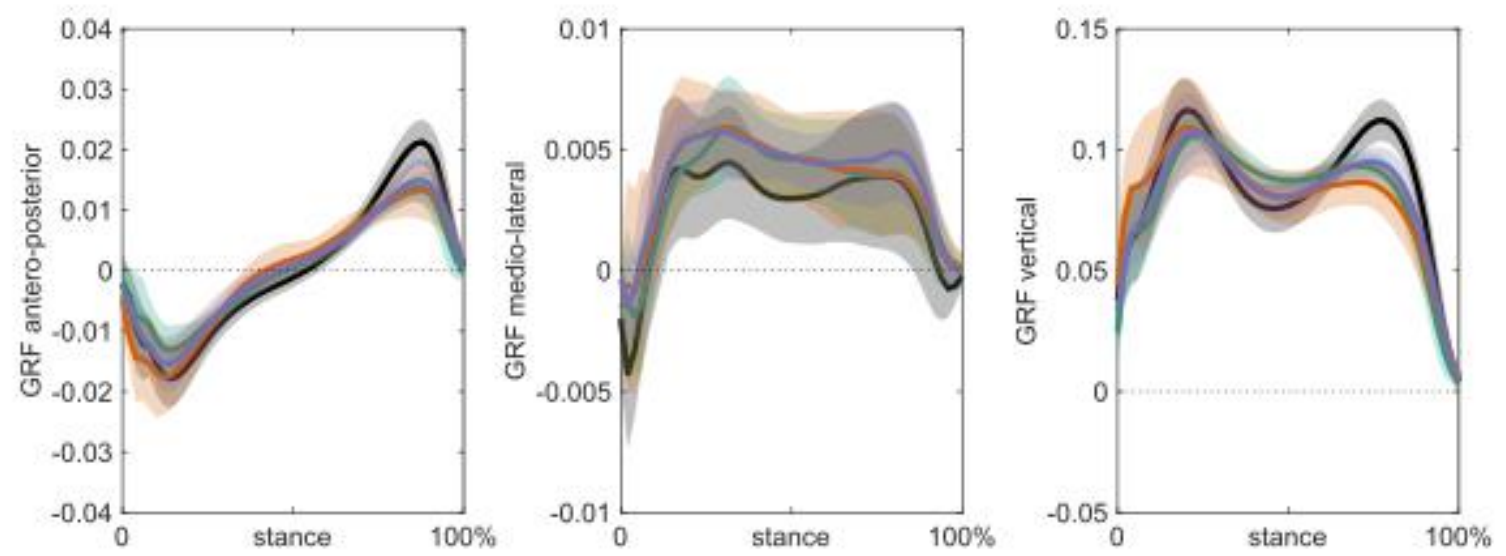

Figure A4: The three components of the ground reaction force (GRF) vector for the three subgroups (colours) and the TD group (black). Normalized for body weight. 